\title{
Dengue Outbreak in Pakistan: Status, Challenges and way Forward
}

\author{
Waris Qidwai
}

This editorial may be cited as: Qidwai W. Dengue Outbreak in Pakistan: Status, Challenges and way Forward. J Liaquat Uni Med Health Sci. 2019;18(04):250-1. doi: 10.22442/jlumhs.191840636

Dengue is vector-borne viral infection transmitted to humans by the female Aedes aegypti mosquito ${ }^{1}$. Infection with dengue virus may result either in an asymptomatic infection or a febrile illness of varying severity ranging from mild illness to more severe forms such as dengue hemorrhagic fever and dengue shock syndrome ${ }^{2}$. At global level, there has been a reported 30 -fold increase in incidence of Dengue in previous five decades ${ }^{1}$.

Recent Dengue outbreak in Pakistan has reached epidemic proportion and has surpassed all previous records. Previous large outbreak occurred in 2011 and affected 27,000 people across the country including 370 deaths. During the current year over 25,000 dengue cases have been confirmed from across the country. This includes 6, 537, 5, 642, 4, 403, 4, 276 2, 750 cases from Islamabad, Punjab, Sindh, Khyber Pakhtun-khwa and Balochistan respectively. Fortunately, deaths reported this year are less than before, mainly because of awareness among public and health care professionals, resulting in earlier diagnosis and treatment of dengue patients ${ }^{3}$.

Reasons for current dengue outbreak in Pakistan are multifactorial. Unfortunately, country lacks an effective functional healthcare delivery system with poor regulatory control over both medical education as well as clinical practice ${ }^{4,5}$. Public health initiatives are severely lacking with poor infrastructure, resulting in lack of mosquito control. Awareness about dengue is lacking among common public with the result that people do not take preventive measures nor seek medical care early enough for better clinical outcomes. Health care professionals are also not trained to properly pick cases early and treat them adequately. Uncontrolled practice of alternative and complimentary medical practitioners, as well as faith healers, promotes non evidence based practices resulting in delay in appropriate treatment of dengue patients at appropriate time. Majority of deaths in dengue are a result of poor hydration among dengue patients resulting in multi organ failure.

We need urgent measures to meet the huge challenge of controlling dengue outbreak. Government should ensure measures are taken to control of mosquito breeding. Public need to be informed about preventive measures to stop breeding of mosquitos. Stagnant water sources need to be identified and removed. Proper nets should be put in place to stop mosquitos from entering homes and work places and places where people gather such as mosques. Effective fumigation should be in place to remove mosquitos. People should be encouraged to wear full sleeve shirts and full trousers and should use mosquito repellents. Such public health measures are much needed and will go a long way in controlling this epidemic.

It's important that print, electronic and social media is used to inform public about the disease, how it spreads and what are its early symptoms for its early detection. It's equally important to educate and train our health care providers how to pick up cases early and how to treat them effectively.

It's important to have required resources to control dengue epidemic. In addition to family doctors, infectious disease experts and nurses, we need diagnostic support as well as advance intensive care services for those with multi organ failure. Kits need to be made available to detect dengue antigen and antibody. Establishment of a functional helpline at government level will help those who need assistance ${ }^{6}$.

Academia has a very important role to control dengue outbreaks. Research into development of effective vaccine $^{7,8}$ along with newer and better diagnostic and treatment modalities is need of the hour. Developing and implementing cost effective models of care that help control dengue epidemic is required. Public private partnership will help ease the crisis because government alone cannot handle the huge challenge of current and recurrent dengue epidemic ${ }^{4}$.

Its time to act fast to control dengue outbreak in the country. All stakeholders should be involved in an effort to bring all available resources together, to prevent dengue associated morbidly and mortality in the country.

\section{REFERENCES}

1. Otu A, Ebenso B, Etokidem A, Chukwuekezie O. Dengue fever- an update review and implications for Nigeria, and similar countries. Afr Health Sci. 2019; 19(2): 2000-2007. doi:10.4314/ahs. v19i2.23.

2. Kosasih H, Alisjahbana B, Nurhayati, de Mast $Q$, Rudiman IF, Widjaja S, et al. The Epidemiology, Virology and Clinical Findings of Dengue Virus Infections in a Cohort of Indonesian Adults in 
Western Java. PLoS Negl Trop Dis. 2016; 10(2): e0004390. doi: 10.1371/journal.pntd.0004390.

3. Junaidi I. Over 25,000 dengue cases reported this year. Dawn Newspaper [Internet]. Published on Oct 11, 2019. Available from: https://www.dawn. com/news/1510207.

4. Qidwai W. Healthcare Delivery and Public Private Partnership in Pakistan: Issues, Challenges and Opportunities. J Liaquat Uni Med Health Sci. 2016; 15(04): 162-3.

5. Ahmad S, Qidwai W. Primary health care reforms in Pakistan: A mandatory requirement for successful healthcare delivery. Middle East J Fam Med. 2016. 14(9): 7-10.

6. Zinger ND, Blomberg SN, Lippert F, Collatz
Christensen H. Satisfaction of 30402 callers to a medical helpline of the Emergency Medical Services Copenhagen: a retrospective cohort study. BMJ Open. 2019; 9: e029801. doi:10.1136/ bmjopen-2019-029801.

7. Perera S, John D, Senanayaka B. Cost effectiveness of dengue vaccination following pre-vaccination serological screening in Sri Lanka. Int J Technol Assess Health Care. 2019 Oct 18: 19. doi: 10.1017/S0266462319000680. [Epub ahead of print]

8. Meissner HC. Complexity in Assessing the Benefit vs Risk of Vaccines: Experience With Rotavirus and Dengue Virus Vaccines. JAMA. 2019; 322 (19): 1861-2. doi:10.1001/jama.2019.16206.

AUTHOR AFFILIATION:

Dr. Waris Qidwai

Professor, Department of Family Medicine Aga Khan University, Karachi, Sindh-Pakistan.

E-mail: waris.qidwai@aku.edu 